\title{
Rotation and torsion of the left ventricle with cardiovascular magnetic resonance tagging: comparison of two analysis methods
}

Lauri Lehmonen ${ }^{1,2^{*}}$ (D, Mikko Jalanko ${ }^{3}$, Mika Tarkiainen $^{4}$, Touko Kaasalainen ${ }^{1}$, Johanna Kuusisto ${ }^{5}$, Kirsi Lauerma ${ }^{1}$ and Sauli Savolainen ${ }^{1,2}$

\begin{abstract}
Background: Left ventricle rotation and torsion are fundamental components of myocardial function, and several software packages have been developed for analysis of these components. The purpose of this study was to compare the suitability of two software packages with different technical principles for analysis of rotation and torsion of the left ventricle during systole.

Methods: A group of hypertrophic cardiomyopathy (HCM) patients ( $N=14$, age $43 \pm 11$ years), mutation carriers without hypertrophy ( $N=10$, age $34 \pm 13$ years), and healthy relatives ( $N=12$, age $43 \pm 17$ years) underwent a cardiovascular magnetic resonance examination, including spatial modulation of magnetization tagging sequences in basal and apical planes of the left ventricle. The tagging images were analyzed offline using a harmonic phase image analysis method with Gabor filtering and a non-rigid registration-based free-form deformation technique. Left-ventricle rotation and torsion scores were obtained from end-diastole to end-systole with both software.

Results: Analysis was successful in all cases with both software applications. End-systolic torsion values between the study groups were not statistically different with either software. End-systolic apical rotation, end-systolic basal rotation, and end-systolic torsion were consistently higher when analyzed with non-rigid registration than with harmonic phase-based analysis $(p<0.0001)$. End-systolic rotation and torsion values had significant correlations between the two software $(p<0.0001)$, most significant in the apical plane.

Conclusions: When comparing absolute values of rotation and torsion between different individuals, softwarespecific reference values are required. Harmonic phase flow with Gabor filtering and non-rigid registration-based methods can both be used reliably in the analysis of systolic rotation and torsion patterns of the left ventricle.
\end{abstract}

Keywords: Cardiovascular magnetic resonance, Tagging, Rotation, Torsion

\footnotetext{
* Correspondence: lauri.lehmonen@helsinki.fi

${ }^{1}$ HUS Medical Imaging Center, Radiology, University of Helsinki and Helsinki

University Hospital, PO Box 340, Fl-00029 Helsinki, HUS, Finland

${ }^{2}$ Department of Physics, University of Helsinki, Helsinki, Finland

Full list of author information is available at the end of the article
}

(C) The Author(s). 2020 Open Access This article is licensed under a Creative Commons Attribution 4.0 International License, which permits use, sharing, adaptation, distribution and reproduction in any medium or format, as long as you give appropriate credit to the original author(s) and the source, provide a link to the Creative Commons licence, and indicate if changes were made. The images or other third party material in this article are included in the article's Creative Commons licence, unless indicated otherwise in a credit line to the material. If material is not included in the article's Creative Commons licence and your intended use is not permitted by statutory regulation or exceeds the permitted use, you will need to obtain permission directly from the copyright holder. To view a copy of this licence, visit http://creativecommons.org/licenses/by/4.0/ The Creative Commons Public Domain Dedication waiver (http://creativecommons.org/publicdomain/zero/1.0/) applies to the data made available in this article, unless otherwise stated in a credit line to the data. 


\section{Background}

Motion of the heart is a result of the order of the arrangement of myofibers in the heart wall. The myofibers are organized into a helical wrap around the left ventricle, with a gradual change in the fiber orientation from a right-handed helix in the subendocardial layers to a left-handed helix in the epicardial layers [1]. As the myofibers contract, the ventricular wall contracts, thickens, and rotates in different planes of the heart. During systole the myofibers create a rotating motion, pushing blood forward and reducing the volume of the left ventricle. When looking at the heart from the apex, this wringing motion is created by a clockwise basal rotation and a counterclockwise apical rotation [2]. Different diseases damage the heart and weaken its motion.

Quantitative analysis is increasingly popular to allow finer distinctions between healthy and diseased tissue and to compare results of different studies more reliably. Cardiovascular magnetic resonance (CMR) tagging is a method for creating temporary visually detectable saturation patterns (stripes or grids) in CMR images. The tagging pattern is created using the same magnetic properties of tissue that are used for image acquisition. During the pulsating motion of the heart the tagging pattern deforms as the heart moves, and by analyzing this deformation, it is possible to determine myocardial motion. Several other imaging methods, such as computed tomography, single photon emission tomography, and positron emission tomography, have also been used for motion analysis of the heart $[3,4]$. The most recent developments have centered around use of ultrasonic methods [5]. Despite advancements, CMR remains the most consistent method and is considered the gold standard in myocardial motion assessment $[6,7]$.

The technique for CMR tagging has existed for over 20 years; however, in clinical work the method is still not widely used due to the lack of easily accessible tools for analysis. Many available analysis tools started as research projects and have later been commercialized. The goal of this work was to investigate the suitability of two available software packages and to compare the results yielded by them in three different study groups: hypertrophic cardiomyopathy (HCM) patients, mutation carriers without hypertrophy, and healthy controls. In HCM, the ventricular wall is asymmetrically thickened, leading to local dysfunction and a change in contractile mechanics. HCM has been employed in this study as a suitable disease model to assess the usefulness of analysis tools for myocardial mechanics.

\section{Methods}

\section{Study population}

The study population was selected from a previous study of myosin-binding protein $\mathrm{C}$ gene (MYBPC), which consisted of 32 patients carrying the Finnish founder mutation in the MYBPC gene (MYBPC3-Q1061X) with left-ventricle hypertrophy consistent with HCM phenotype (left-ventricular maximal wall thickness $>13 \mathrm{~mm}$ in CMR), 15 subjects with the mutation and no HCM phenotype (left-ventricular maximal wall thickness $<13$ $\mathrm{mm}$ in CMR), and their 20 healthy relatives without the mutation [8]. Genetic diagnosis was performed on all subjects [9]. Of these individuals, 36 (14 HCM patients, 10 mutation carriers, and 12 healthy relatives) had undergone CMR with tagging images acquired and were selected for this study. The study protocol was approved by the Ethics Review Board of Helsinki and Uusimaa, and a written informed consent was received from all participants. All of the individuals included in the study underwent a CMR examination between 2009 and 2011 using a Magnetom Avanto $1.5 \mathrm{~T}$ system (Siemens Healthcare, Erlangen, Germany). Six-channel body and six-channel spine coils were used for the acquisition. Prospective ECG-gating and breath-hold were used in all cases to minimize acquisition problems related to arrhythmia and to minimize motion artifacts caused by breathing. The CMR imaging protocol included typical volumetric assessments as well as tagging sequences in basal and apical planes of the left ventricle.

\section{CMR tagging}

In this study, the tagging images were acquired at apical and basal levels of the left ventricle. Different technical solutions exist for the creation of the tagging pattern [10]. The pulse sequence used in all cases in this study was a typical spatial modulation of magnetization tagging sequence [11] provided by the vendor of the magnetic resonance imaging system. The sequence had a grid tagging pattern, $8 \mathrm{~mm}$ slice thickness, $8 \mathrm{~mm}$ distance between the tagging lines, repetition time of 41 $\mathrm{ms}$, echo time of $4.0 \mathrm{~ms}, 14^{\circ}$ flip angle, matrix size of $208 \times 256$, and voxel size of $1.25 \times 1.25 \times 8 \mathrm{~mm}^{3}$. The sequence had 20-25 temporal phases.

\section{Image analysis}

The analysis of the apical and basal tagging images was performed using two different software, Harmonic Phase Flow (HPF) plugin (Computer Vision Center, Barcelona, Spain) [12-14] for Osirix Dicom viewer v7.0.2 (Pixmeo, Geneva, Switzerland) and Segment strain tagging module v2.2 R6190 (Medviso AB, Lund, Sweden) [15, 16]. The two software use different technical approaches in analysis of tagging images. Both software required manual epicardial and endocardial segmentation in a single time frame in the plane of analysis.

One way of analyzing the deformation of the tagging pattern is harmonic phase (HARP) analysis [17]. In HARP analysis, the intensity of a tagged CMR image is 
expressed in complex form in the Fourier space. HARP images are only a material quantity and they remain constant over time. Therefore, they can be used to follow the location of the tagging pattern through the cardiac cycle and to construct a deformation map. The motion following of HARP images is an imageprocessing problem affected by image quality [18]. HPF solves the HARP tracking problem using a Gabor filter bank in an alternative mathematical framework. Optimal results using Gabor filters can be achieved when using different filter parameters in each plane of the left ventricle since the motion of the heart is different in each plane. The motion of a single myocardial point can be quantified by analyzing its motion through the imaging sequence. Rotation is defined as the relative change of an angle $\theta$ between the time points zero and t. The location of a single point of the myocardium at time point $t+1$ is obtained by operating it with a motion vector calculated at time point $t$. The motion of the entire left ventricle is calculated by forming a continuous motion vector (or deformation map) over the imaging sequence. Rotation of the entire left ventricle in a single plane is calculated as a normalized scalar product with respect to the center of mass of the ventricle [19]:

$$
\mathrm{R}(\theta)=\arccos \frac{\mathrm{P}_{0}-\mathrm{C}_{0}, \mathrm{P}_{\mathrm{t}}-\mathrm{C}_{\mathrm{t}}}{\left\|\mathrm{P}_{0}-\mathrm{C}_{0} \mid\right\| \mathrm{P}_{\mathrm{t}}-\mathrm{C}_{\mathrm{t}} \|}
$$

where arccos is the inverse of cosine, and the numerator denotes a vector product between vectors $\mathrm{P}_{0}-\mathrm{C}_{0}$ and $\mathrm{P}_{t}$ $-C_{t} . P_{0}$ and $P_{t}$ are the locations of a single myocardial point at the time points zero and t. $\mathrm{C}_{0}$ and $\mathrm{C}_{\mathrm{t}}$ are the locations of the center of mass of the left ventricle at time points zero and $t$. The denominator is a normalized scalar product of the same vectors. Torsion is then defined as the rotation difference between the apical and basal levels of the left ventricle.

Segment strain tagging module approaches the tagging pattern analysis in image space, working with non-rigid elastic image registration. The tagging module maps the displacement of single myocardial points between consecutive time frames and presents the displacements of all myocardial points as two-dimensional third-order Bspline tensor products [20]. An inter-frame transformation field is then constructed using a limited memory BroydenFletcher-Goldfarb-Shannon optimizer. This transformation field can be used to calculate strains, velocities, and displacements between end-diastole and end-systole of the left ventricle. To solve the displacement or rotation of the leftventricle wall, cumulating the transformation field through all time frames is required [16]. Torsion in Segment software is defined as the difference between apical and basal rotation, normalized with the distance between the two slices, and the mean radius of the heart wall in these slices. Torsion was calculated in the same way as in HPF for better comparison between the two software.

Results of rotation and torsion data were collected, with respect to time, from end-diastole to end-systole with both software, and exported to MATLAB R2019A (The MathWorks, Inc., Natick, MA, USA) for visualization. Additionally, end-systolic values were collected. HPF reports the relative time as a proportion from $0 \%$ (end-diastole) to $100 \%$ (end-systole), while Segment uses an absolute time scale. To visually compare rotation curves derived with each software, Segment curves were manually converted in MATLAB to the same $0-100 \%$ time scale as in HPF.

\section{Statistical analysis and intraobserver variability}

Results are expressed as mean \pm standard deviation. Statistical analysis was performed using IBM SPSS Statistics 25 for Windows (IBM Corp., Armonk, NY, USA). Independent samples Kruskal-Wallis test was used to test for significant differences in volumetric parameters and tagging results, and Spearman's rho was used to test for correlations between rotation and torsion values obtained with the two software. $P$-values less than 0.05 were considered statistically significant. Only endsystolic values were included in the statistical analysis. Bland-Altman method was used to assess intraobserver variability in the tagging results with both software, and all study subjects were selected for the analysis. Mean differences (bias) and 95\% limits of agreement $( \pm 1.96$ standard deviations) were computed.

\section{Results}

\section{Volumetric data}

Baseline clinical information and volumetric data are reported in Table 1 . There were more males in the HCM group and no differences in left-ventricular volumes or ejection fractions between the groups. As expected, the left-ventricular mass was significantly higher in the HCM group.

\section{Rotation and torsion}

The mean analysis time per study was $4 \pm 2$ min in HPF and $5 \pm 2 \mathrm{~min}$ in Segment. All cases were applicable for analysis with both software. End-systolic peak apical rotation, peak basal rotation, and peak torsion values were collected (Table 2). HPF showed constantly smaller values than Segment. Looking at the end-systolic rotation and torsion values obtained with different software, similar observations were detected in the study groups. End-systolic apical rotation was smallest in the HCM group (HPF: $1.8^{\circ} \pm 1.8^{\circ}$; Segment: $3.4^{\circ} \pm 4.5^{\circ}$ ), and endsystolic torsion was largest in the mutation group (HPF: $4.1^{\circ} \pm 1.6^{\circ}$; Segment: $9.5^{\circ} \pm 2.2^{\circ}$ ). The end-systolic rotation 
Table 1 Basic clinical features and volumetric data of each group. Data are presented as mean \pm standard deviation

\begin{tabular}{|c|c|c|c|c|}
\hline Parameter & $\mathrm{HCM} \mathrm{N}=14$ & Mutation $N=10$ & Healthy $N=12$ & $p$-value \\
\hline Number of females (proportion) & $4(25 \%)$ & $10(100 \%)$ & $8(75 \%)$ & - \\
\hline Age (years) & $43 \pm 11$ & $34 \pm 13$ & $43 \pm 17$ & 0.280 \\
\hline Height (cm) & $177 \pm 9$ & $166 \pm 4$ & $169 \pm 9$ & $0.015^{*}$ \\
\hline Weight (kg) & $80 \pm 15$ & $60 \pm 3$ & $79 \pm 11$ & $0.005^{*}$ \\
\hline $\mathrm{BSA}\left(\mathrm{m}^{2}\right)$ & $2.0 \pm 0.7$ & $1.7 \pm 0.0$ & $1.8 \pm 0.3$ & $0.003^{*}$ \\
\hline LVEF (\%) & $63 \pm 8$ & $62 \pm 6$ & $60 \pm 9$ & 0.225 \\
\hline LVEDVI (ml/ m²) & $81 \pm 11$ & $77 \pm 13$ & $79 \pm 12$ & 0.819 \\
\hline LVESVI $\left(\mathrm{ml} / \mathrm{m}^{2}\right)$ & $30 \pm 10$ & $29 \pm 7$ & $32 \pm 11$ & 0.625 \\
\hline LVMI $\left(\mathrm{g} / \mathrm{m}^{2}\right)$ & $60 \pm 18$ & $42 \pm 7$ & $42 \pm 8$ & $0.005^{*}$ \\
\hline Max LV wall thickness (mm) & $22 \pm 7$ & $9 \pm 1$ & $10 \pm 3$ & $<0.0001^{*}$ \\
\hline Distance between tagging planes $(\mathrm{cm})$ & $4.0 \pm 0.5$ & $3.9 \pm 0.3$ & $3.7 \pm 0.2$ & 0.098 \\
\hline
\end{tabular}

BMI Body mass index, BSA Body surface area, LV Left-ventricle, EF Ejection fraction, EDVI End-diastolic volume indexed, ESVI End-systolic volume indexed, MI Mass indexed, ${ }^{*}$ statistically significant $(p<0.050)$

or torsion values were not statistically different between the study groups with either software.

The rotation curves of HCM patients and healthy controls followed similar paths in both apical and basal planes of the left ventricle (Figs. 1 and 2). The largest difference between curves in HPF and Segment was seen in basal rotation in the mutation group (Fig. 2). The other curves agreed well between HPF and Segment. In the apical plane, the end-systolic rotation was higher in mutation carriers than in HCM patients or healthy controls. The rotation curves of mutation carriers were separated from the rotation curves of the other groups in both apical and basal planes. Apical rotation was stronger throughout systole. In Fig. 2, the initial positive basal rotation of mutation carriers was higher than that of healthy controls or HCM patients. Torsion curves of different study groups (Fig. 3) look similar throughout systole.

Absolute rotation and torsion values were significantly different in HPF compared with the respective values in Segment software $(p<0.0001)$. These values were considerably higher in Segment. Statistically significant correlations were detected in apical rotation, basal rotation, and torsion values between these two software (Fig. 4). The correlation in apical rotation was the strongest $(p>0.9)$.

\section{Intraobserver variability}

Bland-Altman plots for both software packages are presented in Fig. 5. Both software packages showed good intraobserver reliability. With HPF, $97 \%$ of data points were within the $95 \%$ confidence interval, compared to 92\% with Segment. Basal rotation showed smaller variability compared to apical rotation with both software. Segment showed slightly smaller bias between two measurements compared to HPF.

\section{Discussion}

The aim of our study was to evaluate the suitability of HPF and Segment software in analyzing rotation and torsion of the left ventricle in different study groups. This is the first study to compare differences between these two software packages. Analysis was successful in all cases and usage of both software was robust. Absolute end-systolic rotation and torsion values differed significantly between the two software, Segment yielding

Table 2 Results for end-systolic rotation and torsion in all study groups

\begin{tabular}{|c|c|c|c|c|}
\hline Parameter & $\mathrm{HCM} \mathrm{N}=14$ & Mutation $N=10$ & Healthy $N=12$ & $p$-value \\
\hline HPF apical rotation $\left({ }^{\circ}\right)$ & $1.8 \pm 1.8$ & $3.0 \pm 1.7$ & $1.9 \pm 1.9$ & 0.266 \\
\hline HPF basal rotation $\left({ }^{\circ}\right)$ & $-1.5 \pm 1.2$ & $-1.1 \pm 0.9$ & $-1.9 \pm 1.3$ & 0.351 \\
\hline HPF torsion $\left({ }^{\circ}\right)$ & $3.3 \pm 1.7$ & $4.1 \pm 1.6$ & $3.8 \pm 1.3$ & 0.358 \\
\hline Segment apical rotation $\left(^{\circ}\right)$ & $3.4 \pm 4.5$ & $6.0 \pm 2.3$ & $4.0 \pm 4.3$ & 0.457 \\
\hline Segment basal rotation $\left(^{\circ}\right)$ & $-3.8 \pm 2.5$ & $-3.6 \pm 2.3$ & $-4.0 \pm 2.5$ & 0.667 \\
\hline Segment torsion $\left(^{\circ}\right)$ & $7.2 \pm 3.7$ & $9.5 \pm 2.2$ & $8.0 \pm 2.6$ & 0.154 \\
\hline Segment torsion, normalized $(\% / \mathrm{mm})$ & $0.17 \pm 0.07$ & $0.22 \pm 0.07$ & $0.22 \pm 0.05$ & 0.195 \\
\hline
\end{tabular}

HCM Hypertrophic cardiomyopathy, HPF Harmonic Phase Flow 

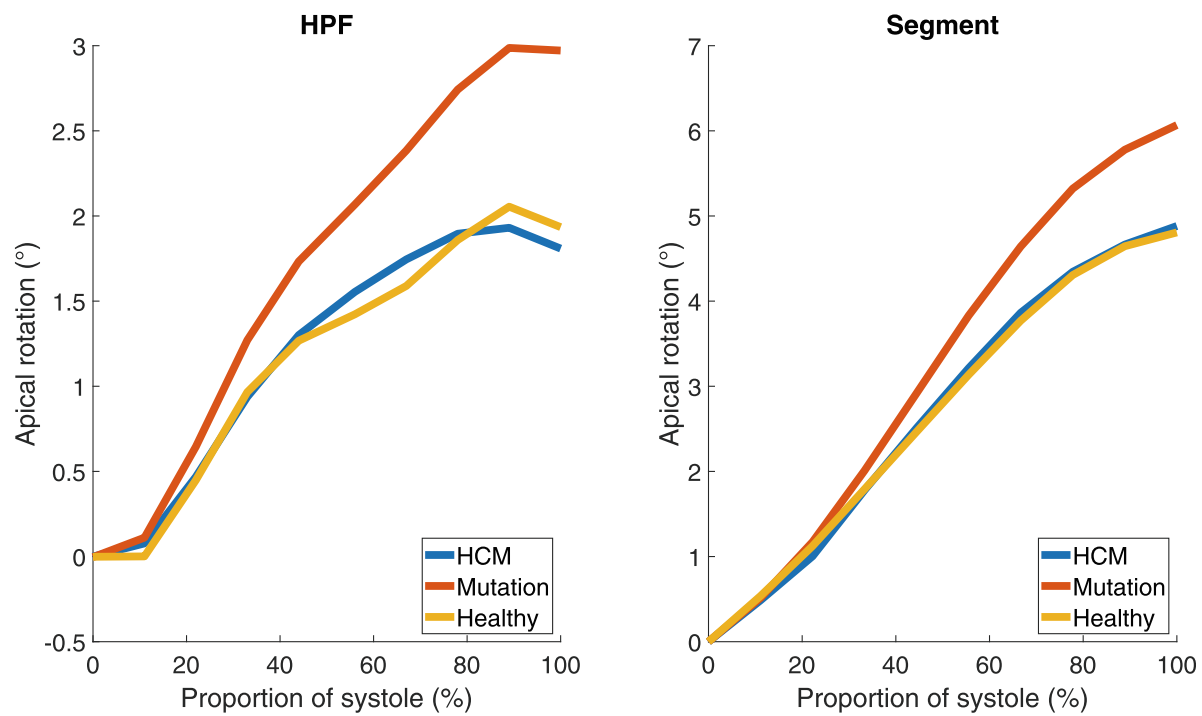

Fig. 1 Mean apical rotation in each study group

systematically larger values. Software-specific reference values are required when comparing absolute values of rotation and torsion between different software. Correlations between the rotation and torsion values obtained with HPF and Segment were statistically significant, the most so in the apical plane.

Based on the results of both software, absolute torsion of the left ventricle did not differ significantly between HCM patients, mutation carriers, and healthy controls. Rotation of the apical level of the left ventricle was increased in mutation carriers. By contrast, basal rotation was slightly lower in this group (Fig. 2). As torsion is calculated as the angle difference between apical and basal rotations, the differences in rotation are not seen in the torsion curves (Fig. 3). This could indicate that HCM mutation without actual hypertrophy might already affect the motion of the left ventricle. However, the sample size is too small to draw definitive conclusions.

The effects of HCM on the motion of the left ventricle have been investigated before, but, as He et al. [21] summarized, the effects of location and extent of hypertrophy on left ventricular function have not attracted much attention, and further studies should be conducted to deepen understanding of disease mechanics. Previous studies have shown that peak torsion in HCM patients can be slightly higher than in control groups [22]. However, in the case of apical hypertrophic cardiomyopathy, apical rotation can be markedly decreased, while basal
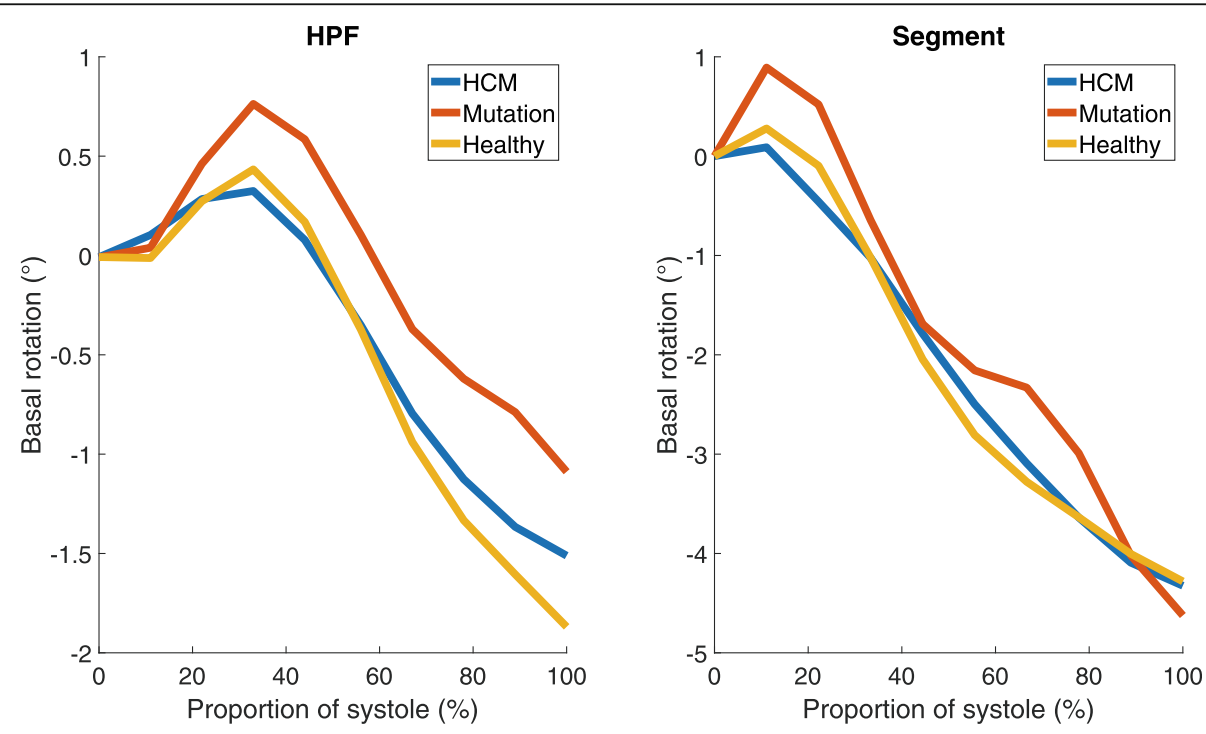

Fig. 2 Mean basal rotation in each study group 

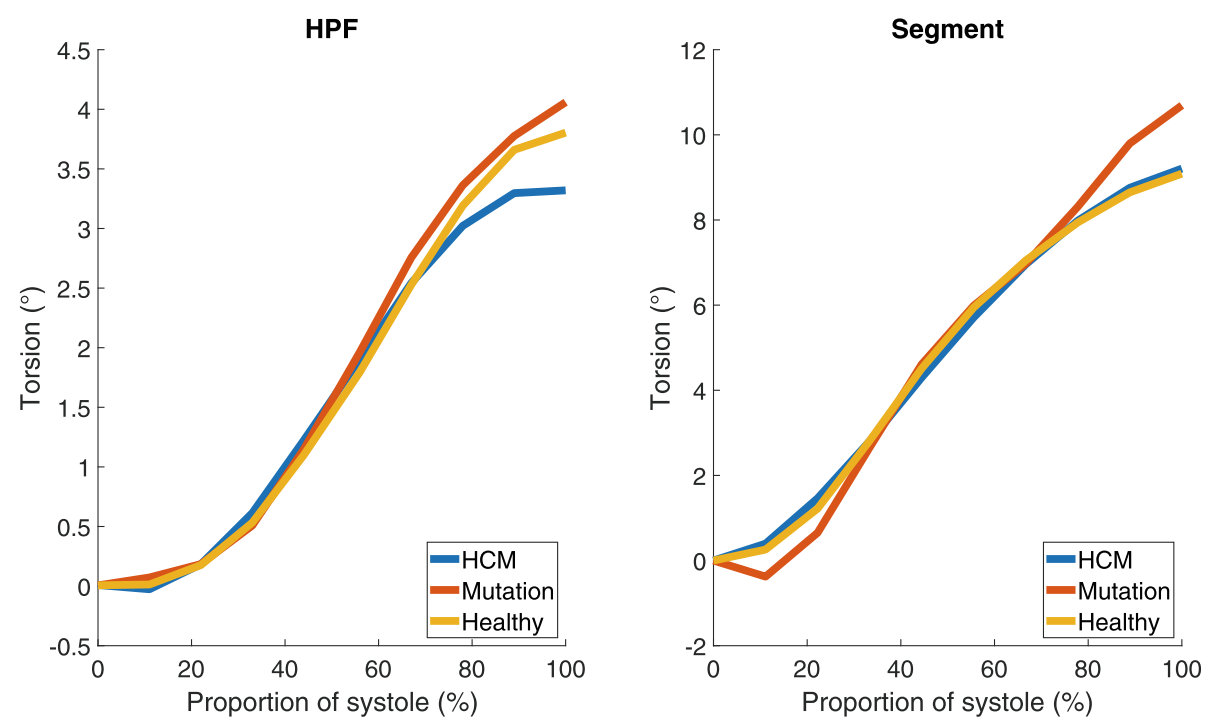

Fig. 3 Mean torsion of the left ventricle in each study group

rotation is preserved [23]. In three of the HCM patients in our study, end-systolic apical rotation was negative, leading to an impaired torsion value. As HCM is a heterogeneous disease with diverse effects on the motion of the heart, more work is needed to elucidate the mechanics of the HCM heart.

Previous studies by Rüssel et al. [24] indicate endsystolic torsion in healthy subjects between basal and apical levels of the left ventricle of $7.7^{\circ} \pm 1.4^{\circ}$. Our result with Segment was similar $\left(8.0^{\circ} \pm 2.6^{\circ}\right)$. However, the result of the HPF was significantly lower $\left(3.8^{\circ} \pm 1.3^{\circ}\right)$. The definition for torsion applied by Rüssel et al. uses the mean radius of apical and basal levels divided by the mean distance between the two planes to normalize the torsion between different-sized hearts. The definition used by HPF for torsion in our work is absolute and does not take into account the size of the heart.

Gabor filter-based motion tracking has been previously shown to result in accurate segmentation of the tagging lines [25]. Gabor filtering achieves optimal locating in both spatial and Fourier domains, making it more suitable for tag line motion analysis than pure HARP [26]. The performance of the HPF algorithm has been assessed by the developers using a computational model

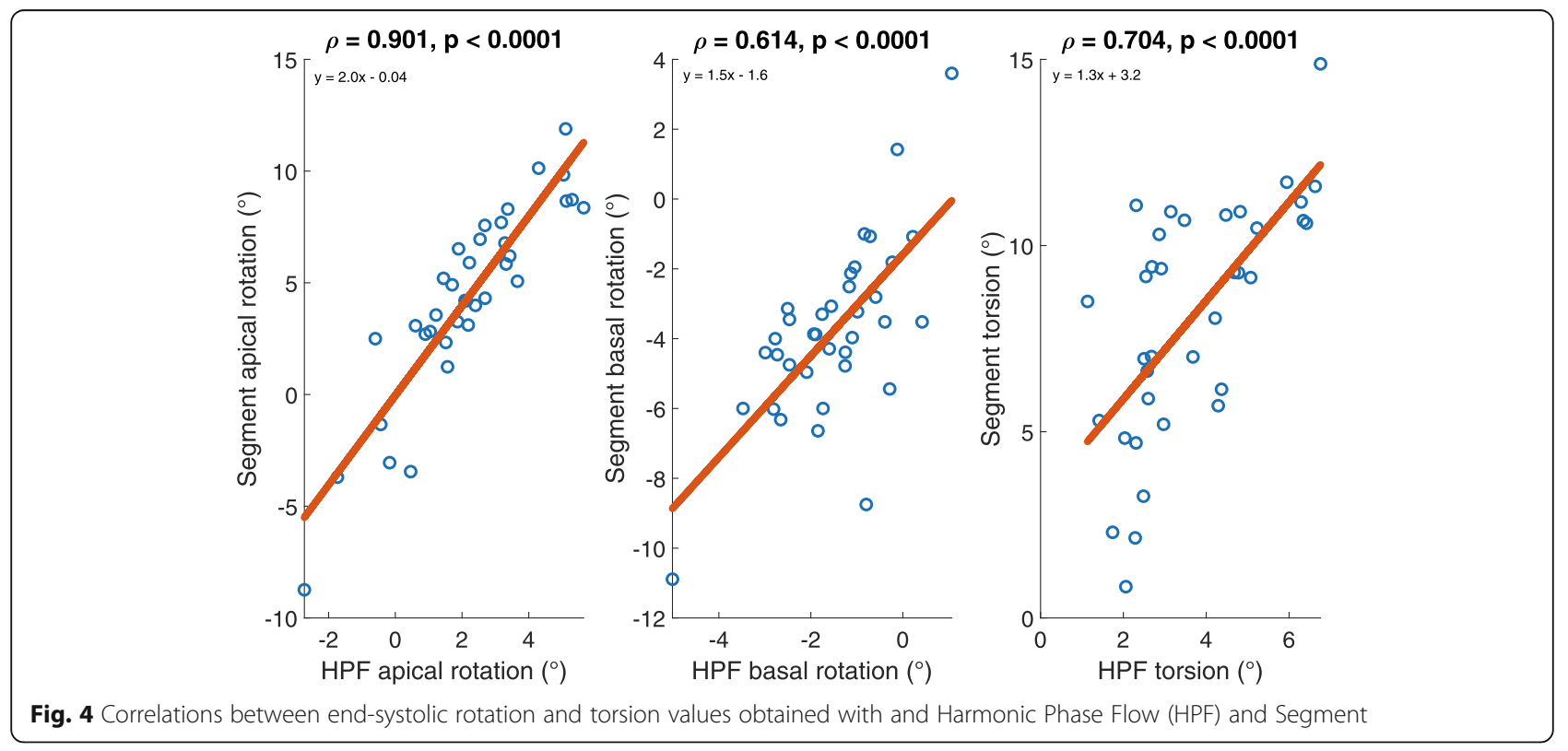




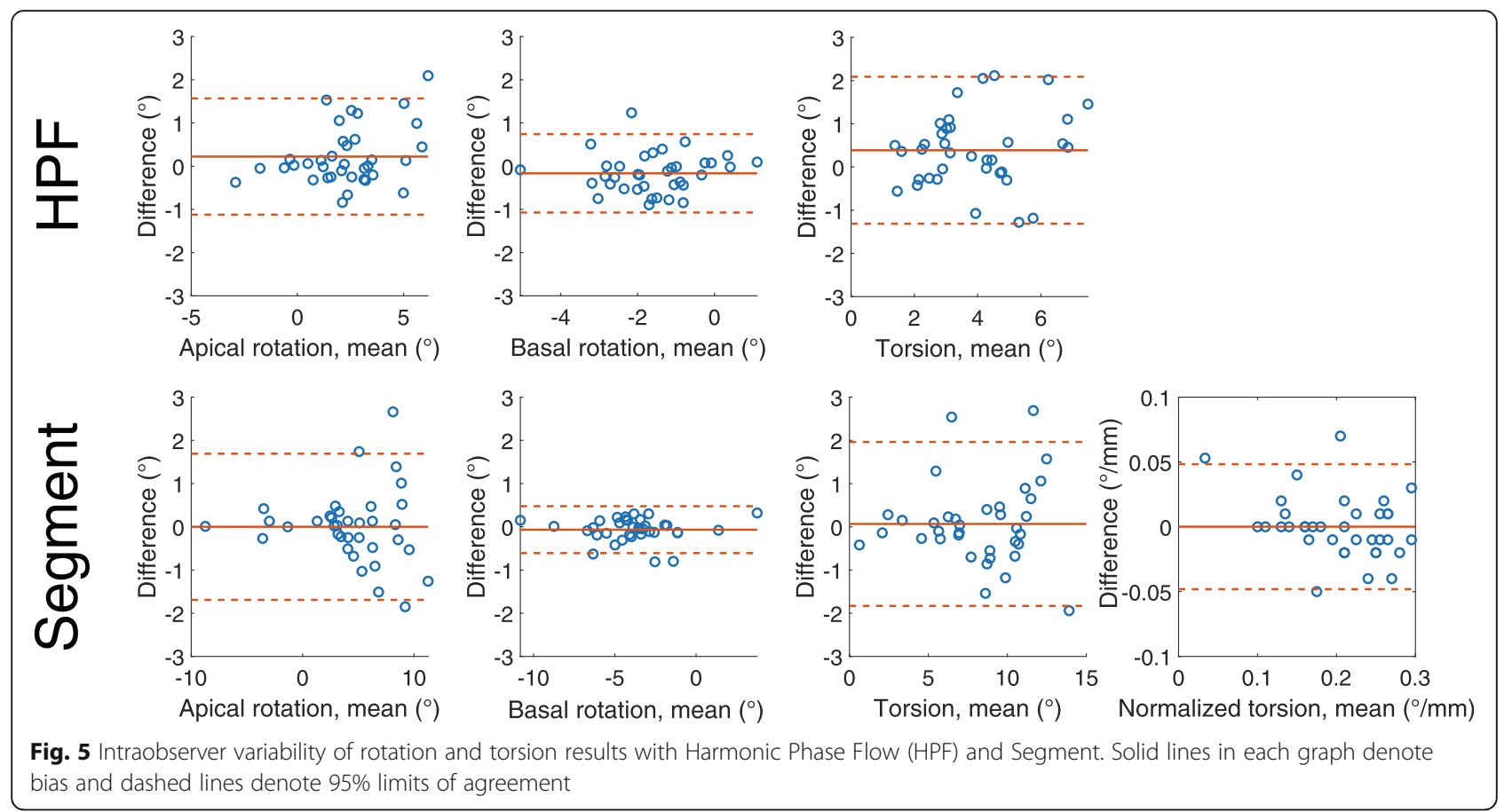

as well as clinical data. They concluded that with sufficient image quality, HPF tracks motion correctly within sub-pixel accuracy [12]. Similarly, Segment strain tagging module has been validated by the developers, and the underlying non-rigid elastic registration-based motion analysis algorithm has been shown to yield clinically reproducible results between different observers with varying level of training $[16,27]$. Our intraobserver analysis of the present study is in line with previous results.

Apical tagging imaging plane should be chosen carefully, as apical rotation is highly dependent on the imaging plane. The generally lower torsion values of HPF in our study could be due to the apical plane being close to midlevel of the left ventricle. Increased end-systolic apical rotation and end-systolic torsion values were detected in subjects with longer distance between the tagging planes.

\section{Limitations}

Our study is limited to the rotation and torsion analyses of systole only, and readers were not blinded to the diagnosis of different cases. Interobserver variability was assessed in the present study. The patient sample of this study, being a subsample of a previous study, is too small to allow definitive conclusions to be drawn regarding the acquired rotation and torsion values between HCM patients, mutation carriers, and their healthy relatives.

\section{Conclusions}

When comparing absolute values of CMR-derived rotation and torsion between different individuals, softwarespecific reference values are required. Harmonic Phase
Flow OsiriX plugin and Segment strain tagging module can both be used to evaluate rotation and torsion patterns during systole, but absolute values between these software are significantly different.

\section{Abbreviations}

CMR: Cardiovascular magnetic resonance; HARP: Harmonic phase; HCM: Hypertrophic cardiomyopathy; HPF: Harmonic Phase Flow; MYBPC: Myosin-binding protein C gene

\section{Acknowledgments}

The authors thank the HCM Study Group of the Universities of Kuopio and Helsinki (Mika Laine, Petri Sipola, Tiina Heliö, Pertti Jääskeläinen) for providing the cardiac CMR imaging data for further analysis.

\section{Authors' contributions}

MJ, MT, JK, and KL designed the study and collected the data. LL MJ, MT, TK, $\mathrm{JK}$, and $\mathrm{KL}$ analyzed and interpreted the data. LL, MJ, TK, and SS wrote the article. The authors read and approved the final manuscript.

\section{Funding}

The study collection of data was supported by the Finnish Medical Foundation, the Finnish Foundation of Cardiovascular Research, the special governmental subsidy for health sciences research of the University Hospital of Kuopio and the Finnish Academy. The analysis of data and writing of the manuscript was supported by HUS Medical Imaging Center.

\section{Availability of data and materials}

The datasets generated and/or analysed during the current study are not publicly available due to the limitations set by the ethical approval for this study. The datasets generated during this study are available from the corresponding author on reasonable request.

Ethics approval and consent to participate

The study was approved by the Ethics Review Board of Helsinki and Uusimaa (HUS 84/2008), and written consent was obtained from all participants.

Consent for publication

Not applicable. 


\section{Competing interests}

The authors declare that they have no competing interests.

\section{Author details}

'HUS Medical Imaging Center, Radiology, University of Helsinki and Helsinki University Hospital, PO Box 340, FI-00029 Helsinki, HUS, Finland. ${ }^{2}$ Department of Physics, University of Helsinki, Helsinki, Finland. ${ }^{3}$ Department of Cardiology, Heart and Lung Center, Helsinki University Hospital and University of Helsinki, Helsinki, Finland. ${ }^{4}$ Department of Clinical Radiology, Kuopio University Hospital, Kuopio, Finland. ${ }^{5}$ Institute of Clinical Medicine, Internal Medicine and Kuopio University Hospital, Kuopio, Finland.

Received: 17 December 2019 Accepted: 19 June 2020

Published online: 01 July 2020

\section{References}

1. Sengupta PP, Korinek J, Belohlavek M, Narula J, Vannan MA, Jahangir A, et al. Left ventricular structure and function: basic science for cardiac imaging. J Am Coll Cardiol. 2006;48(10):1988-2001.

2. Russel IK, Gotte MJ, Bronzwaer JG, Knaapen P, Paulus WJ, van Rossum AC. Left ventricular torsion: an expanding role in the analysis of myocardial dysfunction. JACC Cardiovasc Imaging. 2009;2(5):648-55.

3. Tavakoli V, Sahba N. Cardiac motion and strain detection using 4D CT images: comparison with tagged MRI, and echocardiography. Int J Cardiovasc Imaging. 2014;30(1):175-84.

4. Carla Gilardi M, Rizzo G, Savi A, Landoni C, Bettinardi V, Rossetti C, et al. Correlation of SPECT and PET cardiac images by a surface matching registration technique. Comput Med Imaging Graph. 1998;22(5):391-8.

5. Heyde B, Jasaityte R, Barbosa D, Robesyn V, Bouchez S, Wouters P, et al. Elastic image registration versus speckle tracking for 2-D myocardial motion estimation: a direct comparison in vivo. IEEE Trans Med Imaging. 2013;32(2): 449-59

6. Shehata ML, Cheng S, Osman NF, Bluemke DA, Lima JA. Myocardial tissue tagging with cardiovascular magnetic resonance. J Cardiovasc Magn Reson. 2009:11(55):55

7. Wu L, Germans T, Guclu A, Heymans MW, Allaart CP, van Rossum AC. Feature tracking compared with tissue tagging measurements of segmental strain by cardiovascular magnetic resonance. J Cardiovasc Magn Reson. 2014;16(10):10.

8. Tarkiainen M, Sipola $\mathrm{P}$, Jalanko M, Helio T, Laine $M$, Jarvinen $\mathrm{V}$, et al. Cardiovascular magnetic resonance of mitral valve length in hypertrophic cardiomyopathy. J Cardiovasc Magn Reson. 2016;18(1):33.

9. Jaaskelainen P, Helio T, Aalto-Setala K, Kaartinen M, Ilveskoski E, Hamalainen $\mathrm{L}$, et al. Two founder mutations in the alpha-tropomyosin and the cardiac myosin-binding protein $C$ genes are common causes of hypertrophic cardiomyopathy in the Finnish population. Ann Med. 2013;45(1):85-90.

10. Ibrahim E-S. Myocardial tagging by cardiovascular magnetic resonance: evolution of techniques-pulse sequences, analysis, algoritms, and applications. J Cardiovasc Magn Reson. 2011;13:36.

11. Axel L, Dougherty L. MR imaging of motion with spatial modulation of magnetization. Radiology. 1989;171:841-5.

12. Garcia-Barnés J, Gil D, Pujadas S, Carreras F. A variational framework for assessment of the left ventricle motion. Math Model Nat Phenom. 2008;3(6): $76-100$

13. Garcia-Barnés J, Gil D, Barajas J, Carreras F, Pujadas S, Radeva P. Characterization of ventricular torsion in healthy subjects using Gabor filters and a Variational framework. Comput Cardiol. 2006;33:887-90.

14. Andaluz A, Carreras F, Santa Marta C, Gil D. Myocardial torsion estimation with tagged-MRI in the Osirix platform. In: ISBI workshop on open source medical image analysis software, IEEE; 2012.

15. Heiberg E, Sjögren J, Ugander M, Carlsson M, Engblom H, Arheden H. Desing and validation of segment - a freely available software for cardiovascular image analysis. BMC Med Imaging. 2010;10:1.

16. Morais P, Heyde B, Barbosa D, Queirós S, Claus P, D'Hooge J. Cardiac motion and deformation estimation from tagged MRI sequences using temporal coherent image registration framework. In: Proceedings of the meeting on functional imaging and modelling of the heart (FIMH). London: Lecture notes in Computer Science vol 7945; 2013.

17. Osman NF, McVeigh ER, Prince JL. Imaging heart motion using harmonic phase MRI. IEEE Trans Med Im. 2000;19(3):186-202.
18. Osman NF, Kerwin WS, McVeigh ER, Prince JL. Cardiac motion tracking using CINE harmonic phase (HARP) magnetic resonance imaging. Magn Reson Med. 1999:42(6):1048-60.

19. Carreras F, Garcia-Barnes J, Gil D, Pujadas S, Li CH, Suarez-Arias R, et al. Left ventricular torsion and longitudinal shortening: two fundamental components of myocardial mechanics assessed by tagged cine-MRI in normal subjects. Int J Cardiovasc Imaging. 2012;28(2):273-84.

20. Chandrashekara RMR, Rueckert D. Cardiac motion tracking in tagged MR images using a 4D B-spline motion model and nonrigid image registration. In: 2nd IEEE international symposium on biomedical imaging: Nano to macro; 2004 18-18 April 2004. Arlington: USA IEEE; 2004

21. He X-W, Song Z-Z. Evaluation of left ventricular function, rotation, twist and untwist in patiens with hypertrophic cardiomyopathy. Exp Clin Cardiol. 2013;18(1):e47-e9.

22. Saito M, Okayama $H$, Yoshii T, Hiasa G, Sumimoto T, Inaba S, et al. The differences in left ventricular torsional behavior between patients with hypertrophic cardiomyopathy and hypertensive heart disease. Int J Cardiol. 2011;150(3):301-6.

23. Cheng S-A, Kim H-K, Kim D-H, Kim J-C, Kim Y-J, Kim H-C, et al. Left ventricular twist mechanics in patiens with apical hypertrophic cardiomyopathy: assessment with 2D speckle tracking echocardiography. Heart. 2010;96(1):49-55.

24. Russel IK, Gotte MJ, Kuijer JP, Marcus JT. Regional assessment of left ventricular torsion by CMR tagging. J Cardiovasc Magn Reson. 2008;10(26):26.

25. Qian Z, Montillo A, Metaxas DN, Axel L. Segmenting cardiac MRI tagging lines using Gabor filter banks. Cancun: Proceedings of the 25th Annual International Conference of the IEEE EMBS; 2003.

26. Qian Z, Metaxas DN, Axel L. Extraction and tracking of MRI tagging sheets using a 3D Gabor filter Bank. New York City: Proceedings of the 28th IEEE EMBS Annual International Conference; 2006.

27. Morais P, Marchi A, Bogaert JA, Dresselaers T, Heyde B, D'Hooge J, et al. Cardiovascular magnetic resonance myocardial feature tracking using a non-rigid, elastic image registration algorithm: assessment of variability in a real-life clinical setting. J Cardiovasc Magn Reson. 2017;19(1):24.

\section{Publisher's Note}

Springer Nature remains neutral with regard to jurisdictional claims in published maps and institutional affiliations.
Ready to submit your research? Choose BMC and benefit from:

- fast, convenient online submission

- thorough peer review by experienced researchers in your field

- rapid publication on acceptance

- support for research data, including large and complex data types

- gold Open Access which fosters wider collaboration and increased citations

- maximum visibility for your research: over $100 \mathrm{M}$ website views per year

At $\mathrm{BMC}$, research is always in progress.

Learn more biomedcentral.com/submissions 